\title{
Rupture of ectopic pregnancy in rudimentary horn of uterus at 20 weeks of gestation
}

\author{
Yasmeen Akhtar Haseeb* \\ Assistant Professor/Consultant, College of Medicine, Department of Obstetrics and Gynaecology, University of \\ Dammam Higher Education, Saudi Arabia
}

Received: 05 May 2015

Revised: 06 May 2015

Accepted: 06 June 2015

\section{*Correspondence:}

Dr. Yasmeen Akhtar Haseeb,

E-mail: yhaseeb@uod.edu.sa

Copyright: $\odot$ the author(s), publisher and licensee Medip Academy. This is an open-access article distributed under the terms of the Creative Commons Attribution Non-Commercial License, which permits unrestricted non-commercial use, distribution, and reproduction in any medium, provided the original work is properly cited.

\begin{abstract}
Ectopic pregnancy is a well-known complication in early pregnancy the commonest site is ampullary region of fallopian tube but sometime presentations at rare sites like rudimentary uterine horn can also happen. These rare presentations are difficult to diagnose and needs high index of suspicion on behalf of obstetrician. A 28 years old Saudi female patient with previous cesarean delivery in her second pregnancy at 20 weeks presented to the emergency department of medicine with severe abdominal pain, nausea, vomiting, dizziness and breathlessness. She was investigated on lines of pulmonary embolism but it was all inconclusive. Obstetrics \& gynaecology department was consulted regarding the status of her pregnancy. On examination she was hypotensive and distended abdomen. Clinical and sonographic examination was going with internal bleeding in pelvis. Uterus was seen as separated from the fetus which was floating in free fluid in pouch of Douglas and measurements were going with 20 weeks of gestation. Initial suspicion was scarred ruptured ectopic pregnancy. The patient was immediately shifted to theatre and laparotomy performed with removal of blood and blood clots in abdomen and pelvis, an accessory horn on right side of uterus with a rent on posterior aspect noted and horn was excised. Patient recovered well and received 8 units of packed RBCs, FFP.
\end{abstract}

Keywords: Rudimentary uterine horn, Maternal morbidity mortality, Ectopic pregnancy

\section{INTRODUCTION}

Pregnancy in rudimentary horn of uterus is a rare event which was first reported by Mauriceau in 1969. The reported incidence is 1 in 100000 to 1 in $140000 .{ }^{1}$ It results from incomplete fusion of Mullerian ducts. Despite the recent advances in ultrasound the antenatal diagnosis remains elusive and confirmation of diagnosis is usually made at laparotomy.

Here is a case report of a pregnancy in rudimentary horn of uterus which presented as ruptured non communicating horn. The patient was presented in medical emergency as a case of abdominal pain, vomiting, dizziness and breathlessness.

\section{CASE REPORT}

A 28 years old Saudi female patient with previous cesarean delivery in her second pregnancy at 20 weeks presented to the emergency department of King Fahad Hospital of the University with severe abdominal pain, nausea, vomiting, dizziness and breathlessness. Patient was kept in female observation under medical team with suspicion of pulmonary embolism or gastroenteritis. She was investigated for pulmonary embolism as well. The obstetrics and gynaecological department was consulted regarding her pregnancy. 
On examination patient was pale with cold, clammy skin and anxious looking. Her pulse was 120 beats per minute and blood pressure was $60 / 30 \mathrm{~mm}$ of $\mathrm{Hg}$. Her abdomen was tense, tender and distended. Ultrasound examination was done and there was free fluid all over the abdomen and pelvis .there was a fetus in abdominal cavity floating in fluid there was no bleeding per vaginum and she was very tender on bimanual examination. Our initial suspicion was abdominal pregnancy or ruptured scarred pregnancy.

Patient was taken for laparotomy immediately .Her hemoglobin was 4 gram per deciliter. During laparotomy abdomen and pelvis were found to be full of blood clots which were removed and cavity was cleared. Estimated blood loss was 6 litres. A fetus corresponding to 20 weeks was also removed from pouch of Douglas. Uterus was taken out and a ruptured uterine horn was found on right size, on communicating with the uterus. The horn was excised, hemostasis secured and abdominal cavity was closed .Patient was kept in high dependency units and she was transfused 8 units of packed RBCs. She recovered uneventful and discharged home in good condition. Patient was not counselled in previous cesarean about this uterine anomaly.

\section{DISCUSSION}

Several cases of ruptured pregnancy in uterine horn has been reported in the literature. ${ }^{2}$ This rare uterine anomaly results from failure of fusion of Mullerian ducts. In $83 \%$ to $85 \%$ of cases this rudimentary horn is found to be noncommunicating. ${ }^{3}$ Pregnancy in a non-communicating rudimentary horn occurs through transperitoneal migration of the sperm or fertilized ovum. ${ }^{4}$ It may be associated with rupture, intraperitoneal haemorrhage and intrauterine growth retardation. ${ }^{5}$

Recurrent abdominal pain is the commonest presentation associated with this anomaly during pregnancy. ${ }^{6}$ Most of the cases remain undiagnosed until rupture and the patients present as an acute abdomen or collapsed. A high index of suspicion is required about this catastrophic situation about pregnant patients who present in hospital emergency departments.

\section{CONCLUSION}

The case highlights the importance of considering ruptured ectopic pregnancy in rudimentary horn of uterus by detailed history, clinical examination and involvement of senior colleagues in order to reduce maternal morbidity and mortality.

This case also highlights the importance of surveying uterus, tubes, ovaries while performing a cesarean section. Any abnormality found during this survey must be documented in patients' notes and counseling of patient with future plan of management must be conveyed to her postoperatively to avoid this ongoing tragedy of mother hood.

Funding: No funding sources

Conflict of interest: None declared

Ethical approval: Not required

\section{REFERENCES}

1. Holden R, Hart P. First trimester rudimentary horn pregnancy: a prerupture ultrasound diagnosis. Obstet Gynaecol. 1983;61:565-85.

2. Nahum GG. Rudimentary Uterine horn pregnancy: a case report on surviving twins delivered 8 days apart. J Reprod Med. 1997;42:525-32.

3. Heinonen PK. A unicornuate uterus and a rudimentary horn. Fertil Steril. 1997;68:224-30.

4. Panayotidis C, Abdel-Fattah M, Leggots M. Rupture of rudimentary horn of a unicornuate uterus at 15 weeks of gestation. J Obstet Gynaecol. 2004;24:3234.

5. Shin JW, Kim HJ. A case of live birth in a noncommunicating rudimentary horn of uterus. J Obstet Gynaecol Res. 2005;31:329-31.

6. Falcon T, Gidwani G, Paraiso M, Beverly C, Goldberg J. Anatomical Variations in rudimentary horn of unicornuate uterus: implications for laparoscopic surgery. Hum Reprod. 1997;12:263-5.

Cite this article as: Haseeb YA. Rupture of ectopic pregnancy in rudimentary horn of uterus at 20 weeks of gestation. Int J Reprod Contracept Obstet Gynecol 2015;4:1209-10. 